\title{
Cultivo del nematodo Panagrellus redivivus (Goodey, 1945) en un medio de avena enriquecida con Spirulina sp.
}

\author{
Nematode culture of Panagrellus redivivus (Goodey, 1945) \\ with Spirulina sp. - enriched oatmeal \\ Ramón de Lara ${ }^{1}$, Thalía Castro ${ }^{1}$, Jorge Castro ${ }^{1}$ y Germán Castro ${ }^{1}$ \\ ${ }^{1}$ Universidad Autónoma Metropolitana-Xochimilco. Departamento El Hombre y su Ambiente. Laboratorio de Alimento Vivo \\ Calzada del Hueso No. 1100. Col. Villa Quietud. México, 04960, D.F. \\ rlara@correo.xoc.uam.mx
}

\begin{abstract}
The nematode Pangrellus redivivus was cultured in two media: one with oat flakes and the other with Spirulina sp.-enriched oat flakes, in $15 \times 15 \times 5 \mathrm{~cm}$ plastic containers with $200 \mathrm{~g}$ oat flakes and $300 \mathrm{~mL}$ purified water. Five grams of Spirulina was used in the medium. SYSTAT version 10.2 was used for statistical analysis; to determine the significant differences between treatment a unidirectional analysis of variance (ANOVA) was used with a confidence level of $\alpha=0.05$. The results show that growth of the nematode population in the Spirulina-enriched medium presented the highest abundance of individuals on the second week of culture, whereas the population grown in the oat flakes medium showed the highest abundance on the fifth week of culture and did not reach the number of organisms attained by the population cultured in the Spirulina-enriched medium. The amino acids content of the populations from both media were compared to those reported for Artemia fed with Spirulina, observing that the amounts were higher for most amino acids in $P$. redivivus cultured in the Spirulinaenriched medium. The composition of fatty acids in the nematode cultures in both media depicted significant differences for the linoleic, arachidonic, and eicosapentaenoic fatty acids, which were found in a higher percentage than reported for $P$. redivivus cultures in oat flakes supplemented with sunflower oil. These data reveal that the cyanobacterium Spirulina sp. accelerates growth of the nematode populations and allows the presence of amino and fatty acids, making these nematodes suitable to cover the nutritional requirements of freshwater fish larvae, but further studies are needed to demonstrate it.
\end{abstract}

Key words: Panagrellus, culture, aminoacids, fatty acids, oat, Spirulina
Resumen.- El nematodo Panagrellus redivivus se cultivó en dos medios: uno con hojuelas de avena y otro con hojuelas de avena enriquecido con Spirulina sp., en recipientes de plástico de $15 \times 15 \times 5 \mathrm{~cm}$ de altura con $200 \mathrm{~g}$ de hojuela de avena y $300 \mathrm{~mL}$ de agua purificada. Se utilizaron $5 \mathrm{~g}$ de Spirulina en el medio. Se utilizó el programa SYSTAT versión 10.2 para el análisis estadístico; para determinar las diferencias significativas entre los tratamientos, se usó un análisis de varianza unidireccional (ANDEVA) con una confiabilidad $\alpha=0,05$. Los resultados obtenidos muestran que el crecimiento de las poblaciones de nematodos en el medio enriquecido con Spirulina presentó la mayor abundancia de individuos a la segunda semana del cultivo, mientras que la población que creció en el medio de avena, presentó su mayor registro a la quinta semana del cultivo y no alcanzó el número de organismos que tuvo la población cultivada en el medio con Spirulina. El contenido de aminoácidos de las poblaciones de ambos medios se comparó y se cotejaron con los reportados para Artemia alimentada con Spirulina, observándose que en la mayoría de los aminoácidos las cantidades fueron mayores en $P$. redivivus cultivado en el medio con Spirulina. La composición de ácidos grasos en los nematodos cultivados en ambos medios presentó diferencias significativas entre los ácidos grasos linoleico, araquidónico y eicosapentenoico los cuales fueron en mayor porcentaje que los reportados en $P$. redivivus cultivado en avena con aceite de girasol. Estos datos demuestran que la cianobacteria Spirulina sp. acelera el crecimiento de la población del nematodo y permite la presencia de aminoácidos y ácidos grasos, y hace que estos nematodos puedan cubrir los requerimientos nutritivos de larvas de peces dulceacuícolas, sin embargo, se requieren estudios que lo demuestren.

Palabras clave: Panagrellus, cultivo, aminoácidos, ácidos grasos, avena, Spirulina 


\section{Introducción}

Las etapas larvales de los organismos acuáticos, en su mayoría, requieren de alimento vivo al inicio de su alimentación exógena; los organismos más comunes que se utilizan en la acuicultura, como alimento vivo son microalgas, rotíferos y nauplios de Artemia (Santiago et al. 2004) siendo Artemia la más empleada (Lavens \& Sorgeloos 2000), sin embargo, en la acuicultura se requiere tener una mayor gama de organismos que sirvan como alimento vivo, que suplan las necesidades nutricias de los depredadores y que sean de fácil adquisición y bajos costos. Varios estudios han demostrado que el nematodo Panagrellus redivivus es un alimento adecuado para las primeras etapas larvarias de peces y crustáceos (Kahan \& Appel 1975, Kahan et al. 1980, Wilkenfeld et al. 1984, Biedenbach et al. 1989, Kumlu \& Fletcher 1997, Kumlu et al. 1998, Ricci et al. 2003, Santiago et al. 2003, Santiago et al. 2004, Schlechtriem et al. 2004b). No obstante que se ha demostrado que este nematodo es excelente fuente de alimento, su utilización no ha sido tan amplia como debiera debido a problemas de producción en alta escala; al respecto, existe el trabajo de Ricci et al. (2003) quienes señalan métodos para producir grandes masas de $P$. redivivus, a bajo costo; también el trabajo de Schlechtriem et al. (2002) quienes siguieron el método de Ricci et al. (2003) y señalan que obtuvieron producciones de 400 millones de nematodos por kilogramo de medio de cultivo.

En la actualidad se realizan varias investigaciones con diferentes métodos para la producción masiva del nematodo P. redivivus (Schlechtriem et al. 2002). También se hacen experimentos con diferentes medios de cultivo, sabiendo que éstos tienen una influencia directa en la composición nutrimental del nematodo y que pueden modificar su composición bioquímica, (Rouse et al. 1992); unos ejemplos son los trabajos de Kumlu \& Fletcher (1997) quienes enriquecieron el medio con los ácidos grasos eicosapentaenoico y docohexaenoico y el pigmento asthaxantina y consideraron a $P$. revivivus como una buena dieta para las larvas de Penaeus indicus; Schlechtriem et al. (2004c) demostraron que enriqueciendo el medio avena con ácidos grasos indispensables, $P$. redivivus puede ser un alimento determinado para una especie en particular. El medio más utilizado para crecer $P$. redivivus es el de avena (Santiago et al. 2003) aunque también se emplean las harinas de trigo y de maíz
(Radwin \& Rouse 1990) y la levadura Saccharomyces cerevisae (Ricci et al. 2003, Schlechtriem et al. 2004b, Schlechtriem et al. 2005). Por otra parte, Spirulina ha sido utilizada como un enriquecedor en la composición de aminoácidos y ácidos grasos; como ejemplo, son las investigaciones reportadas con esta cianofita por Cuzon et al. (1981), quienes la utilizaron como ingrediente en la formulación de dietas para Penaeus japonicus; Matsuno et al. (1979) consideraron el alto contenido de carotenoides que tiene Spirulina sp. para intensificar el color de la carpa de ornato. Otro crustáceo que ha mostrado beneficios al ingerir Spirulina sp. es Artemia (Person-Le Ruyet 1976, Castro 1993 y Castro et al. 1994).

El objetivo de este trabajo fue el evaluar la influencia de la adición de Spirulina en el medio de cultivo de avena en relación al crecimiento, composición y concentración de aminoácidos y ácidos grasos de la población de $P$. redivivus bajo condiciones naturales de laboratorio.

\section{Material y métodos}

\section{Panagrellus redivivus}

El nematodo $P$. redivivus fue proporcionado por el Acuario de Veracruz, Veracruz y se ha conservado, en cultivo, en el laboratorio de Alimento Vivo de la UAMunidad Xochimilco.

\section{Alimentos para $P$. redivivus}

Los ingredientes que se emplearon para preparar el medio de cultivo fueron: hojuelas de avena comercial; Spirulina sp. en polvo y agua purificada.

\section{Cultivo}

Para el cultivo se utilizaron seis recipientes plásticos de $15 \times 15 \mathrm{~cm}$ y $5 \mathrm{~cm}$ de altura. En cada recipiente se colocaron $200 \mathrm{~g}$ de hojuelas de avena y $300 \mathrm{~mL}$ de agua purificada. En tres recipientes, además se agregó a cada uno, $5 \mathrm{~g}$ de Spirulina sp. en polvo, y con una espátula, se mezclaron los ingredientes. Para evitar que las esporas de hongos proliferaran en los medios, los recipientes se esterilizaron en un horno de microondas, a máxima potencia, durante 5 minutos. Luego de dejar enfriar, a todos los recipientes se les agregaron $200 \mathrm{~mL}$ de agua purificada para hidratar los medios, quedando un espesor de $2 \mathrm{~cm}$ en cada recipiente. 
La siembra de $P$. redivivus se hizo agregando $5 \mathrm{~g}$ de este organismo, en cada recipiente y mezclándolos en todo el medio para que quedaran totalmente distribuidos, luego, éstos se cubrieron con una malla de $0,36 \mathrm{~mm}$ para evitar la contaminación por insectos. El cultivo se mantuvo, durante nueve semanas, a temperatura ambiente entre 19 y $22^{\circ} \mathrm{C}$, con un $\mathrm{pH}$ entre 2,6 y 3,2; el cultivo se revisó diariamente y solo se repuso, con agua purificada, el líquido perdido por evaporación, volviéndose a mezclar para mantener el medio homogéneo.

\section{Crecimiento de la población}

El registro del crecimiento de la población se realizó cada semana, en cada uno de los recipientes; se recolectó en 10 sitios de la superficie del medio de cultivo, y se tomó una muestra de $0,10 \mathrm{~g}$. en cada sitio.

Los organismos recolectados de cada recipiente, se colocaron en viales y se fijaron en alcohol a $70^{\circ}$ y unas gotas solución de lugol, el cual servía tanto para fijar como para colorear a los organismos y facilitar la cuenta. Mediante una pipeta Pasteur, se tomó de cada vial una muestra de $0,10 \mathrm{~g}$ la cual se colocó en portaobjetos, distribuyéndola a lo largo de los mismos a manera de frotis, para evitar que se concentraran los ejemplares en un sitio y de este modo, facilitar su cuantificación mediante un contador manual. La observación se hizo a través de un microscopio estereoscópico Olimpus ZX40 con objetivo de 20X. La cuenta se realizó durante las nueve semanas que se mantuvo el cultivo.

\section{Análisis químicos}

Para los análisis químicos, al final de las nueve semanas del experimento, se recolectaron los nematodos mediante el tamizado (1,54 - 0,30 y 0,05 $\mathrm{mm}$ de luz de malla) del medio de cada recipiente y se lavaron con agua corriente. En el último tamiz quedaron los nematodos con la menor cantidad de partículas de avena y se colocaron en vasos de precipitado de $250 \mathrm{~mL}$ para separarlos por decantación; esta operación se hizo tres veces y se filtraron a través de tela con luz de malla de $0,03 \mathrm{~mm}$; posteriormente se secaron en una estufa con temperatura constante $\left(40^{\circ} \mathrm{C}\right)$ durante 24 horas.

\section{Análisis de aminoácidos}

El análisis de aminoácidos se efectúo en una muestra seca de un gramo de $P$. redivivus proveniente de las tres repeticiones de ambos cultivos. Las determinaciones se hicieron mediante cromatografía de líquidos de alta presión, en resina de intercambio catiónico, diluida con un gradiente de amortiguadores de $\mathrm{pH}$ de 3,1 a 5,6 y de $0,2 \mathrm{M}$ a 2,0 $\mathrm{M}$ de citrato (Beckman 1985), en un analizador Beckman System Gold 6300 automático.

\section{Análisis de ácidos grasos}

La cuantificación de ácidos grasos se realizó mediante la extracción de lípidos totales con la técnica de Soxhlet (Rubinson \& Rubinson 1998). La muestra obtenida se sometió a una metanólisis ácida (metanoltolueno-ácido sulfúrico en proporción de 30:15:1) para obtener los ácidos grasos metilados, siguiendo el método descrito por Minnikin et al. (1980).

Los ácidos grasos (ésteres metílicos FAME) fueron inyectados en un cromatógrafo de gas Varian Start $3380 \mathrm{CP}$ con una columna capilar DB-23 de $30 \mathrm{~m} \mathrm{x}$ $0,53 \mathrm{~mm}$. El gas Helio fue usado como eluyente. La temperatura del inyector fue de $170^{\circ} \mathrm{C}$, y la del detector de $300^{\circ} \mathrm{C}$. Para la identificación de los ácidos grasos se utilizó un estándar Supelco 37 componente FAME Mix.

\section{Análisis estadístico de los datos}

El tratamiento estadístico de los datos se realizó con el programa estadístico SYSTAT versión 10.2, mediante el cual se realizó la estadística descriptiva: determinaciones de la media y la desviación estándar; determinación de las diferencias significativas entre los distintos tratamientos, utilizando un análisis de varianza unidireccional (ANDEVA) (Triola, 2000), con una confiabilidad $\alpha=0.05$.

\section{Resultados}

\section{a) Crecimiento poblacional}

En la Tabla 1 se presentan los datos promedio de nematodos por gramo y por semana de cada medio de cultivo. 
Tabla 1

\section{Comparación del número de organismos $\mathrm{g}^{-1}$ de Panagrellus redivivus por semana, en los dos cultivo: Avena sola y Avena enriquecida con Spirulina sp.}

Comparison of the number of organisms $\mathrm{g}^{-1}$ of Panagrellus redivivus per week, in the two cultures: oat alone and oat enriched with Spirulina sp.

\begin{tabular}{clccccc}
\hline Semana de cultivo & \multicolumn{5}{c}{ Medio de cultivo } \\
\cline { 2 - 5 } & 30570 & $\pm 7440^{\mathrm{a}}$ & 25120 & $\pm 2800^{\mathrm{a}}$ \\
\hline 1 & 53520 & $\pm 2590^{\mathrm{a}}$ & 194790 & $\pm 27910^{\mathrm{b}}$ \\
2 & 66020 & $\pm 3770^{\mathrm{a}}$ & 152940 & $\pm 9450^{\mathrm{b}}$ \\
3 & 113380 & \pm & $\pm 610^{\mathrm{a}}$ & 79830 & $\pm 1370^{\mathrm{b}}$ \\
4 & 143660 & \pm & $\pm 020^{\mathrm{a}}$ & 62860 & $\pm 2180^{\mathrm{b}}$ \\
5 & 65110 & $\pm 1900^{\mathrm{a}}$ & 46950 & $\pm 3770^{\mathrm{b}}$ \\
6 & 32530 & $\pm 380^{\mathrm{a}}$ & 27640 & $\pm 2440^{\mathrm{a}}$ \\
7 & 12560 & $\pm 1510^{\mathrm{a}}$ & 13220 & $\pm 830^{\mathrm{a}}$ \\
8 & 47850 & $\pm 9070^{\mathrm{a}}$ & 9150 & $\pm 770^{\mathrm{b}}$ \\
\hline
\end{tabular}

Los valores en las desviaciones estándar con diferentes letras indican diferencias significativas $(\mathrm{P}<0.05)$.

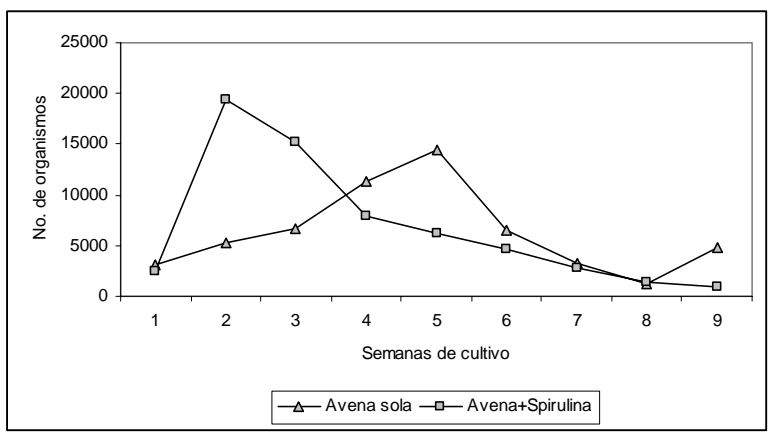

Figura 1

Comparación del número de organismos producidos de Panagrellus redivivus en dos medios de cultivo: Avena sola y Avena enriquecida con Spirulina sp.

Comparison of the number of organisms $\mathrm{g}^{-1}$ of Panagrellus redivivus per week, in the two cultures: oat alone and oat enriched with Spirulina sp.

En la segunda semana se puede observar una diferencia significativa en el crecimiento de la población, en donde el medio enriquecido con Spirulina alcanza a producir 194.790 nematodos $\mathrm{g}^{-1}$. En cuanto al medio con avena sola, es hasta la quinta semana que el número de nematodos $\mathrm{g}^{-1}$ alcanza su densidad máxima con 143.660 organismos. En ambos medios de cultivo la población decrece después de obtener su valor máximo (Fig.1).

\section{b) Aminoácidos}

En la Tabla 2 se presentan el perfil del los aminoácidos registrados y los valores de la concentración de cada uno de ellos en Panagrellus redivivus, obtenidos en el cultivo de avena sola y avena enriquecida con Spirulina.

Del análisis estadístico realizado a los datos con lo aminoácidos, no se observaron diferencias significativas entre los valores de ambos medios. En la misma Tabla se incluyen los datos reportados por Schlechtriem et al. (2004a), quienes cultivaron a esta especie con avena sola y avena enriquecida con aceite de girasol. También en la misma Tabla se compara el contenido de aminoácidos en Artemia alimentada con Spirulina que reportaron Castro et al. (1994). 
Tabla 2

Comparación del perfil de aminoácidos (g 100 $\mathrm{mg}^{-1}$ peso seco) en Panagrellus redivivus cultivado en diferentes medios y de Artemia alimentada con Spirulina

Comparison of the amino acids profile (g 100 $\mathrm{mg}^{-1}$ dry weight) in Panagrellus redivivus cultivated in different media and Artemia fed with Spirulina

\begin{tabular}{|c|c|c|c|c|c|}
\hline \multirow[b]{2}{*}{ Aminoácidos } & \multicolumn{5}{|c|}{ Medio de cultivo } \\
\hline & Avena sola & Avena sola* & $\begin{array}{l}\text { Avena enriquecida } \\
\text { con Spirulina }\end{array}$ & $\begin{array}{c}\text { Avena } \\
\text { enriquecida con } \\
\text { aceite de girasol* }\end{array}$ & $\begin{array}{c}\text { Artemia } \\
\text { alimentada con } \\
\text { Spirulina } * * \\
\end{array}$ \\
\hline Aspártico & 25.20 & 10.70 & 33.83 & 10.70 & $\mathrm{Nd}$ \\
\hline Treonina & 7.52 & 4.30 & 9.11 & 4.40 & 0.97 \\
\hline Serina & 8.59 & 4.30 & 10.19 & 4.20 & $\mathrm{Nd}$ \\
\hline Glutámico & 60.72 & 19.90 & 65.69 & 20.30 & $\mathrm{Nd}$ \\
\hline Glicina & 19.30 & 5.40 & 20.49 & 5.60 & $\mathrm{Nd}$ \\
\hline Alanina & 30.58 & 4.90 & 30.61 & 5.20 & $\mathrm{Nd}$ \\
\hline Cisteina & 1.07 & 1.50 & 1.52 & 1.40 & 0.07 \\
\hline Valina & 17.59 & 4.90 & 19.45 & 5.00 & 0.57 \\
\hline Metionina & 0.19 & 1.30 & 4.17 & 1.10 & 0.64 \\
\hline Isoleucina & 6.29 & 4.00 & 9.53 & 4.20 & 1.33 \\
\hline Leucina & 14.63 & 6.60 & 17.28 & 6.70 & 1.37 \\
\hline Tirosina & 1.16 & 4.20 & 1.42 & 3.20 & 0.90 \\
\hline Fenilalanina & 8.64 & 4.30 & 10.18 & 5.00 & 1.00 \\
\hline Histidina & 25.78 & 4.20 & 25.20 & 4.50 & 0.76 \\
\hline Lisina & 7.94 & 7.30 & 11.77 & 6.80 & 1.46 \\
\hline Arginina & 2.27 & 5.00 & 9.16 & 5.00 & 1.57 \\
\hline
\end{tabular}

\section{C) Ácidos grasos}

En la Tabla 3 se presenta el perfil de los ácidos grasos esenciales del Panagrellus redivivus en los dos cultivo y se observan diferencias significativas entre las concentraciones del ácido linoleico (18:2w6); araquidónico (20:2w6) y eicosapentanoico (20:5w3). También se incluyen los datos reportados por Schlechtriem et al. (2004a) con el fin de realizar la comparación.

\section{Discusión}

La diferencia en la producción de $P$. redivivus cultivado en avena sola y avena con Spirulina es aproximadamente de $26 \%$ en un tiempo de tres semanas. Este incremento es posible que se deba a la influencia de Spirulina en la producción de microorganismos, que son el alimento de los nematodos y que favorecen el crecimiento de la población. Sin embargo, es necesario realizar estudios microbiológicos para establecer las diferencias en cuanto al tipo y número de microorganismos en los diferentes medios.

De acuerdo a lo anterior, es recomendable utilizar a esta cianofita con el fin de producir alimento en poco tiempo y en gran volumen para alimentar larvas de peces. Por el contrario, cuando solo se requiere mantener un cultivo de este nematodo, el medio con avena sola puede ser la opción para el mantenimiento del nematodo. 
Tabla 3

Composición de ácidos grasos (\% del total de ácidos grasos) en Panagrellus redivivus en diferentes medios de cultivo

Fatty acids composition (\% of the total fatty acids) in Panagrellus redivivus in different cultures

\begin{tabular}{|c|c|c|c|c|}
\hline \multirow[t]{2}{*}{ Composición de ácidos grasos } & \multicolumn{4}{|c|}{ Medio de cultivo } \\
\hline & Avena sola* & Avena sola & $\begin{array}{c}\text { Avena enriquecida } \\
\text { con Spirulina }\end{array}$ & $\begin{array}{l}\text { Avena enriquecida con } \\
\text { aceite de girasol* }\end{array}$ \\
\hline \multicolumn{5}{|l|}{ Ácidos grasos saturados } \\
\hline $14: 0$ & $0,5 \pm 0,0$ & $0,32 \pm 0,06^{\mathrm{a}}$ & $0,50 \pm<0,01^{\mathrm{a}}$ & $0,6 \pm 0,0$ \\
\hline $16: 0$ & $10,2 \pm 0,5$ & $18,03 \pm 4,33^{\mathrm{a}}$ & $19,58 \pm 0,28^{\mathrm{a}}$ & $10,7 \pm 0,9$ \\
\hline $18: 0$ & $6,3 \pm 0,1$ & $2,49 \pm 0,07^{\mathrm{a}}$ & $2,52 \pm 0,12^{\mathrm{a}}$ & $5,4 \pm 0,6$ \\
\hline \multicolumn{5}{|l|}{ Ácidos grasos monoinsaturados } \\
\hline $16: 1 n-7$ & $1,4 \pm 0,1$ & $0,59 \pm 0,16^{\mathrm{a}}$ & $0,70 \pm 0,05^{\mathrm{a}}$ & $2,1 \pm 0,1$ \\
\hline $18: 1 n-9$ & $13,3 \pm 0,5$ & $32,67 \pm 0,51^{\mathrm{a}}$ & $26,26 \pm 0,33^{\mathrm{a}}$ & $14,0 \pm 0,8$ \\
\hline $18: \ln -7$ & $7,8 \pm 0,0$ & $3,88 \pm 1,16^{\mathrm{a}}$ & $3,93 \pm 0,57^{\mathrm{a}}$ & $6,2 \pm 0,3$ \\
\hline $20: 1 n-9$ & $0,6 \pm 0,0$ & $2,10 \pm 0,01^{\mathrm{a}}$ & $1,48 \pm 0,13^{\mathrm{a}}$ & $0,4 \pm 0,0$ \\
\hline \multicolumn{5}{|l|}{ n-6 PUFA } \\
\hline $18: 2 \mathrm{n}-6$ & $24,0 \pm 0,3$ & $25,66 \pm<0,01^{\mathrm{a}}$ & $22,30 \pm 0,57^{\mathrm{b}}$ & $36,0 \pm 3,1$ \\
\hline $18: 3 n-6$ & $1,0 \pm 0,1$ & $0,11 \pm<0,01^{\mathrm{a}}$ & $0,127 \pm 0,02^{\mathrm{a}}$ & $0,8 \pm 0,1$ \\
\hline $20: 2 n-6$ & $2,1 \pm 0,0$ & $0,29 \pm 0,01^{\mathrm{a}}$ & $0,85 \pm<0,01^{\mathrm{b}}$ & $2,1 \pm 0,1$ \\
\hline $20: 3 n-6$ & $6,8 \pm 0,1$ & $1,30 \pm<0,01^{\mathrm{a}}$ & $1,73 \pm 0,12^{\mathrm{a}}$ & $4,2 \pm 0,5$ \\
\hline $20: 4 n-6$ & $11,1 \pm 0,6$ & $0,81 \pm<0,01^{\mathrm{a}}$ & $0,70 \pm 0,07^{\mathrm{a}}$ & $5,6 \pm 0,5$ \\
\hline \multicolumn{5}{|l|}{ n-3 PUFA } \\
\hline $20: 5 n-3$ & $2,3 \pm 0,0$ & $0,56 \pm 0,09^{\mathrm{a}}$ & $1,73 \pm 0,12^{b}$ & $1,7 \pm 0,2$ \\
\hline $22: 6 n-3$ & $\mathrm{Nd}$ & $0,08 \pm<0,01^{\mathrm{a}}$ & $0,05 \pm<0,01^{\mathrm{a}}$ & $\mathrm{Nd}$ \\
\hline
\end{tabular}

* Datos tomados de: Schlechtriem et al, (2004a),

Desviación estándar en la misma fila, con diferente letra tienen diferencias significativas $(\mathrm{P}<0,05)$,

$\mathrm{Nd}=$ no determinado

Al comparar los resultados del cultivo de avena sola y de avena enriquecida con Spirulina, con lo reportado por Ricci et al. (2003), quienes utilizaron avena sola y avena enriquecida con aceite de girasol, aireación y una concentración de sal del $8 \%$ en 750 g de medio, los valores de producción con avena sola fue 241,333 nematodos $\mathrm{g}^{-1}$, los obtenidos en este trabajo, con avena sola fueron 143,660 nematodos $\mathrm{g}^{-1}$ lo que equivale a un $40,47 \%$ de diferencia. En lo que respecta al cultivo de avena enriquecido con aceite de girasol, estos autores obtuvieron 333,333 individuos $\mathrm{g}^{-1}$, a diferencia de los 194,790 nematodos $\mathrm{g}^{-1}$ que se obtuvieron en el cultivo de avena enriquecida con Spirulina que equivaldría a un $41,56 \%$ menos. Probablemente, la diferencia en la producción esté establecida por la aireación y la salinidad, así como por las condiciones axénicas en el laboratorio. Sin embargo, es conveniente señalar que uno de los propósitos del presente trabajo, es el de proporcionar una alternativa de producción de alimento vivo mediante una técnica sencilla, que pueda adaptarse a condiciones prácticas y económicas de cultivo que requieren productores piscícolas de regiones con escaso desarrollo tecnológico y económico.

En cuanto a los aminoácidos, los valores encontrados en este estudio son mayores que los informados por Schlechtriem et al. (2004a), con excepción de metionina, tirosina y arginina. En cuanto a la avena enriquecida con Spirulina, en todos los casos la concentración sobrepasa considerablemente a los datos obtenidos con los nematodos enriquecidos con aceite de girasol, con excepción de la cisteina y de la tirosina que presentan una concentración mayor.

Comparando el contenido de aminoácidos de Artemia alimentada con Spirulina con los obtenidos en 
$P$, redivivus se puede apreciar que las concentraciones en el nematodo son mayores que las reportadas para Artemia.

Schlechtriem et al. (2004c) mencionan que $P$. redivivus posee ácidos grasos saturados así como la actividad metabólica necesaria para sintetizar ácidos grasos insaturados, utilizando a los lípidos de cadena corta como precursores de los ácidos $\mathrm{C}_{16}, \mathrm{y}_{18}$, aunque se observa que la actividad de desaturación se da principalmente para los ácidos $\mathrm{C}_{16}$. El perfil de ácidos grasos de $P$. redivivus se ve influenciado tanto por la composición de éstos en el medio de cultivo y la capacidad metabólica del organismo para sintetizarlos (Santiago et al. 2004, Schlechtriem et al. 2004c). Al comparar los resultados de los tratamientos de avena sola y avena enriquecida con Spirulina, contra los datos reportados por Schlechtriem et al. (2004a), en medios de cultivo de avena sola y avena con aceite de girasol, se encontraron mayores cantidades de los ácidos oleico

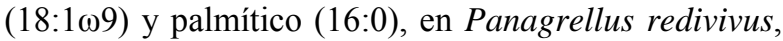
en el medio enriquecido con Spirulina y se puede deducir que esta cianoficia es la responsable de este incremento. Es por esto que ambos ácidos grasos influyen en la composición nutritiva de $P$. redivivus y lo hace ser un alimento vivo adecuado para la alimentación de alevines de peces principalmente dulceacuícolas.

\section{Conclusiones}

La presencia de Spirulina en el medio de cultivo es capaz de acelerar el crecimiento de la población en dos semanas de cultivo.

No es recomendable continuar el cultivo con el medio enriquecido con Spirulina después de la segunda semana, ya que la población de nematodos decrece.

Si el propósito del cultivo de $P$. redivivus es solo de mantenimiento, se deberá utilizar un medio de avena sola hasta la quinta semana.

El contenido de aminoácidos y ácidos grasos en ambos cultivos se encuentra presente para cubrir los requerimientos nutritivos de larvas de peces dulceacuícolas.

Es necesario realizar bioensayos con larvas de peces y crustáceos alimentados con $P$. redivivus cultivado en un medio de avena enriquecido con Spirulina para evaluar su crecimiento y supervivencia.

\section{Agradecimientos}

Agradecemos las observaciones y sugerencias de los evaluadores que enriquecieron el presente trabajo.

\section{Literatura citada}

Biedenbach JM, LL Smith, TK Thomsen \& AL Lawrence. 1989. Use of the nematode Panagrellus redivivus as an Artemia replacement in larval penaeid diet. Journal of the World Aquaculture Society 20 (2): 6171.

Castro BT. 1993. Biología y cultivo de Artemia franciscana en el Ex Lago de Texcoco, de Ecatepec, Estado de México, Tesis doctoral, Facultad de Ciencias, Universidad Nacional Autónoma de México, 72 pp.

Castro BT, AR De Lara \& MJ Castro. 1994. Artemia franciscana alimentada con Spirulina fresca, como dieta de especies acuáticas comerciales. Revista Hidrobiológica 4 (1-2): 15-20.

Cuzon G, R Dos-Santos, M Hew \& G Poullaouac. 1981. Use of Spirulina in Shrimp (Penaeus japonicus) diet. Journal of the World Mariculture Society 12(2): 282-291.

Kahan D \& L Appel. 1975. The value of Pangrellus sp, (Nematoda) as food for fish. $10^{\text {th }}$ European Symposium on Marine Biology, Ostend, Belgium, 1: 243-253.

Kahan D, T Bar-El, Y Brandstein, M Rigbi \& B Olano. 1980. Free living nematodes as a dietary supplement in the rearing of fish fry and hatcheries. General Fisheries Council for the Mediterranean Studies and Reviews 57: 67-78.

Kumlu M \& DJ Fletcher. 1997. The nematode Panagrellus redivivus as an alternative live feed for larval Penaeus indicus. Israeli Journal of Aquaculture-Bamidgeh 49 (1): 12-18.

Kumlu MD, J Fletcher \& CM Fisher. 1998. Larval pigmentation, survival and growth of Penaeus indicus feed the nematode Panagrellus redivivus enriched with astaxanthin and various lipids. Aquaculture Nutrition 4: 193-200.

Lavens P \& P Sorgeloos. 2000. The history, present status and prospects of the availability of Artemia cyst for aquaculture. Aquaculture 181: 397-403.

Matsuno TS, M Nagata, T Iwahashi, M Koike \& M Okada. 1979. Intensification of color of fancy red carp with zeaxanthin and myxoxanthophyll, major carotenoid 
constituents of Spirulina. Bulletin of the Japanese Society of Scientific Fisheries 45(5): 627-632.

Minnikin ED, GI Hutchinson \& BA Caldicott. 1980. Thinlayer chromatography of methanolysates of mycolic acidcontaining bacteria. Journal of Chromatography 188: 221233.

Person-Le Ruyet J. 1976. Elevage larvaire d' Artemia salina (Branchiopode) sur nurriture inerte: Spirulina maxima (Cyanophycee). Aquaculture 8: 157-167.

Radwin IA \& DB Rouse. 1990. Yield characteristics of the free-living nematode Panagrellus redivivus in different culture media. The Progressive Fish Culturist 52(4): 237240 .

Ricci M, AP Fifi, A Ragni, C Schlechtriem \& U Focken. 2003. Development of a low cost technology for mass production of the free-living nematode Panagrellus redivivus as an alternative live food for first feeding fish larvae. Applied Microbiology and Biotechnology 60: 556-559.

Rouse BD, CD Webster, IA Radwin. 1992. Enhancement of the fatty acid composition of the nematode Panagrellus redivivus using three different media. Journal of the World Aquaculture Society 23(1): 89- 95.

Rubinson FJ \& AK Rubinson. 1998. Química analítica contemporánea, 664 pp. Ed. Prentice May, México.

Santiago CB, M Ricci, A Reyes-Lampa. 2004. Effect of nematode Panagrellus redivivus density on growth, survival, feed consumption and carcass composition of bighead carp Artistichthys nobilis (Richardson) larvae. Journal of Applied Ichthyology 20: 22-27.

Santiago CB, AC Gonzal, M Ricci \& S Harpaz. 2003. Response of bighead carp Artistichthys nobilis and Asian catfish Clarias macrocephalus larvae to free-living nematode Panagrellus redivivus as alternative feed. Journal of Applied Ichthyology 19: 239-243.
Schlechtriem C, U Focken, M Ricci \& K Becker. 2001. Testing of nematodes Panagrellus redivivus grown on different media as alternative live food for first feeding carp Cyprinus carpio larvae. $6^{\text {th }}$ Asian Fisheries Forum Book of Abstracts, 24 pp.

Schlechtriem C, M Ricci, U Focken \& K Becker. 2002. A new technology for the mass production of the free-living nematode Panagrellus redivivus, as alternative live food for first-feeding fish larvae: a contribution to the improvement of sustainable seed supply for aquaculture. International Symposium, Sustaining Food Security and Managing Natural Resources in Southeast Asia. Challenges for the $21^{\text {st }}$ Century. January 8-11, 2002, Chiang Mai, Thailand.

Schlechtriem C, M Ricci, U Focken \& K Becker. 2004a. The suitability of the free-living nematode Panagrellus redivivus as live food for first-feeding fish larvae. Journal of Applied Ichthyology 20: 161-168.

Schlechtriem C, M Ricci, U Focken \& K Becker, 2004b. Mass produced nematodes Panagrellus redivivus as live food for rearing carp larvae: preliminary results. Aquaculture Research 35: 547-551.

Schlechtriem C, DR Tocher, JR Dick \& K Becker. 2004c. Incorporation and metabolism of fatty acids by desaturation and elongation in the nematode, Panagrellus redivivus. Nematology (6): 783-795.

Schlechtriem C, U Focken \& K Becker. 2005. Digestion and assimilation of the free-living nematode Panagrellus redivivus fed to first feeding coregonid larvae: Evidence from histological and isotopic studies. Journal of the World Aquaculture Society 36(1): 24-31.

Triola FM. 2000. Estadística elemental, 791 pp. $7^{\mathrm{a}}$ ed, Addison Wesley Longman, México.

Wilkenfield JS, AL Lawrence \& FD Kuban. 1984. Survival, metamorphosis and growth of penaeid shrimp larvae reared on a variety of algal and animal foods. Journal of the World Mariculture Society 15: 31-49. 